\title{
Low back related leg pain - An investigation of construct validity of a new classification system
}

Authors: Axel GM Schäfer $\mathrm{PhD}^{\S, 1}$, Toby M Hall $\mathrm{PhD}^{2,3}$, Roman Rolke MD ${ }^{4}$, RolfDetlef Treede MD, Prof. ${ }^{5}$, Kerstin Lüdtke MSc ${ }^{6}$, Joachim Mallwitz MD ${ }^{6}$, Kathryn N Briffa PhD, Prof. ${ }^{2}$

${ }^{1}$ HAWK University of Applied Sciences and Arts, Hildesheim, Germany

${ }^{2}$ School of Physiotherapy, Curtin Innovation Health Research Institute, Curtin University of Technology Perth, Australia

${ }^{3}$ Manual Concepts, Perth, Australia

${ }^{4}$ Department of Palliative Medicine, University of Bonn, Germany

${ }^{5}$ Ruprecht-Karl University, Heidelberg, Germany

${ }^{6}$ Rückenzentrum am Michel, Hamburg, Germany

${ }^{\S}$ Corresponding author

Corresponding author's address:

Axel Schäfer

Bismarckstr. 99

20253 Hamburg

Phone: $\quad+494043280274$

Mobile: $\quad+491787317016$

Email: $\quad$ axel.schaefer@hawk-hhg.de 
Abstract

2 Background: Leg pain is associated with back pain in $25-65 \%$ of all cases and

3 classified as somatic referred pain or radicular pain. However, distinction between the

4 two may be difficult as different pathomechanisms may cause similar patterns of pain.

5 Therefore a pathomechanism based classification system was proposed, with four

6 distinct hierarchical and mutually exclusive categories: Neuropathic Sensitization

7 (NS) comprising major features of neuropathic pain with sensory sensitization;

8 Denervation (D) arising from significant axonal compromise; Peripheral Nerve

$9 \quad$ Sensitization (PNS) with marked nerve trunk mechanosensitivity; and

10 Musculoskeletal (M) with pain referred from musculoskeletal structures.

11 Objective: To investigate construct validity of the classification system

12 Methods: Construct validity was investigated by determining the relationship of 13 nerve functioning with subgroups of patients and asymptomatic controls. Thus

14 somatosensory profiles of subgroups of patients with low back related leg pain

15 (LBRLP) and healthy controls were determined by a comprehensive quantitative

16 sensory test (QST) protocol. It was hypothesized that subgroups of patients and

17 healthy controls would show differences in QST profiles relating to underlying 18 pathomechanisms.

19 Results: 77 subjects with LBRLP were recruited and classified in one of the four

20 groups. Additionally, 18 age and gender matched asymptomatic controls were

21 measured. QST revealed signs of pain hypersensitivity in group NS and sensory

22 deficits in group D whereas Groups PNS and M showed no significant differences

23 when compared to the asymptomatic group. 
1 Conclusions: These findings support construct validity for two of the categories of

2 the new classification system, however further research is warranted to achieve

3 construct validation of the classification system as a whole.

4

5 Keywords: Low back pain, Leg pain, Classification system, Validity, Quantitative

$6 \quad$ Sensory Testing, QST

7 


\section{1. Introduction}

2 Low back related leg pain (LBRLP) is common with up to $65 \%$ of patients with low

3 back pain reporting accompanying leg pain [1,2]. These cases account for a

4 disproportionately large amount of the costs of medical care and disability

5 compensation caused by low back pain (LBP) [3] as leg pain is associated with more

6 severe pain and disability outcomes [4]. Traditionally, LBRLP is classified as somatic

7 referred pain ("pseudoradicular pain”) or projected radicular pain [5]. However,

8 despite advanced diagnostic technology, the distinction between these two entities

9 remains difficult as different structures in the lower back can evoke similar patterns of

10 pain. Pain radiating as far as the toes can stem from intervertebral disks,

11 zygapophyseal joints, muscles, and fascia in addition to the lumbar nerve roots [6-8].

13 Randomized controlled trials investigating the effectiveness of conservative treatment

14 of patients with radiating leg pain show inconsistent findings $[9,10]$. One explanation

15 for this could be the failure to correctly classify subjects into homogenous treatment-

16 specific subgroups, with consequent lack of effect due to inappropriate treatment.

17 There are recommendations from the pain literature that for the more complex pain

18 conditions related to nerve injury a classification system based on pathomechanisms

19 offers greater diagnostic and treatment value and may also provide information about

20 the prognosis and natural course of the disorder [11].

22 In order to refine the differentiation of radicular and pseudoradicular pain and hence

23 gain treatment efficacy, we introduced a new mechanism based classification system

24 [12] based on the original classification proposed by Elvey and Hall [13]. The aim of

25 this system is to improve treatment outcome, particularly with respect to identifying 
1 patients most likely to respond to neural mobilization. Depending on the assumed

2 predominance of pathomechanisms, LBRLP is classified into four distinct subgroups.

3 Prioritized, these categories are (Figure 1):

19 This new classification system has demonstrated good interrater reliability with $\kappa=$ $200.72[14]$ and has shown prognostic ability [15]. The objective of the present study 21 was to investigate construct validity of the classification system by determining the relationship of diagnostic groups with the results from Quantitative Sensory Testing 23 (QST) [16]. 


\section{2. Methods}

2 Study design and hypotheses

3 This observational, cross-sectional study was designed to investigate construct

4 validity of a new classification system for subjects with LBRLP. Construct validity is

5 based on testing hypotheses about relationships of the instrument under study (i.e. the

6 classification system) with other instruments measuring similar constructs [16]. The

7 construct measured both by the instrument under study (i.e. the classification system)

8 and the reference instrument (QST) is pain mechanisms. We tested the hypothesis that

9 QST parameters will differ between subgroups of subjects with LBRLP and a group

10 of asymptomatic subjects.

11 Ethical approval

12 This study was approved by the Human Research Ethics Committee of the Curtin

13 University of Technology. All patients provided written informed consent prior to 14 participating in the study.

15 Subjects and recruitment

16 Subjects were recruited at a multidisciplinary pain clinic in Hamburg, Germany. 162

17 consecutive patients with LBRLP referred for physiotherapy at the clinic were

18 screened for eligibility. To be considered for inclusion subjects were required to be

19 between 18 and 75 years of age, with unilateral LBRLP of more than 6 weeks

20 duration. Exclusion criteria were history of lower quadrant surgery or trauma within

21 the past 6 months, nerve root block within the past four weeks, other neuropathic

22 pathology such as diabetes or polyneuropathies, vascular disease in the lower

23 extremities, inflammatory arthropathies, contraindications to manual therapy

24 techniques and inability to understand written / spoken German. Of the 162 subjects 
1 screened, 77 were eligible and willing to participate (Figure 2). Another 18 age and

2 gender matched healthy volunteers were recruited as control subjects to provide

3 normative data for z-score standardization of QST parameters into standard deviation

4 units for comparison.

\section{$5 \quad$ Quantitative sensory testing (QST)}

6 A comprehensive battery of QST devices that was developed and validated by the

7 German Research Network on Neuropathic Pain [17] was used as the reference

8 instrument.

10 This QST battery tests all relevant submodalities of the somatosensory system.

11 Seven tests are used to measure 13 parameters consisting of thermal pain thresholds

12 for cold and hot stimuli; thermal detection thresholds for the perception of cold, warm

13 and thermal sensory limen ${ }^{1}$; paradoxical heat sensations; mechanical pain thresholds

14 for pinprick and blunt pressure; mechanical detection thresholds for touch and

15 vibration; a stimulus-response-function for pinprick sensitivity; dynamic mechanical

16 allodynia for stroking light touch; as well as pain summation to repetitive pinprick

17 stimuli. Thus QST evaluates the function of sensory nerve fibres and their respective

18 pathways [18] by analysing multiple parameters of sensory testing. Thus obtained

19 sensory profiles of patients may exhibit whether dominant features of sensory deficit

20 (loss of function) or sensory hyperexcitability (gain of function) exist, indicative for

21 specific pain mechanisms [19, 20].

23 The test protocol has been shown to have good test-retest and inter-tester reliability

\footnotetext{
${ }^{1}$ Thermal sensory limen is the difference in sensory threshold between alternating cold and warm stimuli
} 
1 [21] as well as acceptable concurrent validity [22-24].

3 We tested three body regions; the lower back, the dorsum of the foot and the dorsum

4 of the hand. In subjects with LBRLP test sites were within the painful region of the

5 back and on the dorsum of the affected foot. A site remote to the painful regions

6 (dorsum of the ipsilateral hand) was also tested, as changes in the somatosensory

7 system associated with chronic pain have also been reported in body areas remote to

8 the source of pain. It has been shown that these changes manifest in negative signs

9 such as hypoesthesia [25] as well as in positive signs such as pain sensitivity to blunt

10 pressure [26]. The ipsilateral hand was always tested first, followed alternately by foot

11 or back in patient groups. In the control group, testing of the different areas was

12 conducted alternately.

\section{Classification}

14 All symptomatic subjects were classified into one of four groups following a pre-

15 established examination protocol [12] (Figure 1). The assessment protocol includes

16 subjective questions relating to area of pain, duration of symptoms, and aggravating

17 and easing factors. The subjective components of the LANSS questionnaire [27] were

18 incorporated into the subjective assessment to screen for predominantly positive

19 symptoms indicative of sensitization of the somatosensory system. The physical

20 examination included a neurological examination to screen for motor and sensory

21 deficits, neural tissue provocation tests (straight leg raise test; prone knee bend test,

22 active flexion test in standing, nerve palpation) [13] and the objective components for

23 the total LANSS score (altered pin prick sensation and light touch allodynia). 
1 The classification system as a whole has demonstrated good inter-rater reliability [14]

2 as well as predictive ability [15]. The LANSS has demonstrated good discriminate

3 validity [27].

5 Subjects scoring 12 or more on the LANSS scale were classified as NS. The LANSS

6 questionnaire was designed to detect pain of predominantly neuropathic origin, a cut

7 off score of $\geq 12$ is indicative for a likely contribution of neuropathic pain mechanisms

8 to the patients pain [27]. Mechanisms underlying neuropathic pain may be both

9 central or peripheral [28], however items within the LANSS scale are primarily

10 concerned with identifying positive features of neuropathic pain, such as hyperalgesia

11 and allodynia in areas distant to the lesion which are hall mark signs for central pain 12 mechanisms $[29,30]$.

14 In our earlier papers [12, 14] we referred to the group with a LANSS score $\geq 12$ as

15 "Central Sensitization". In retrospect, this was not the most appropriate term and was 16 probably misleading. In the present paper we refer to the group with a LANSS scale $\geq$ 1712 as "Neuropathic Sensitization". "Neuropathic" to more adequately reflect the 18 construct of the LANSS scale and "Sensitization" as the LANSS tests primarily for 19 positive signs indicative for gain of function. The only item within the LANSS testing 20 for negative signs is the test for altered pin-prick sensation.

22 Subjects scoring less than 12 on the LANSS scale and with at least two or more

23 positive tests in two of four different categories: reflexes; muscle power; light touch;

24 or pinprick sensitivity [14] were classified as "Denervation". We chose the term 
1 "Denervation" as it encompasses both ventral (efferent) and dorsal (afferent) root

2 dysfunction.

4 Subjects in group PNS are characterized by positive nerve provocation tests [31] with

5 a LANSS score $<12$ and in the absence of marked neurological deficits. The term

6 "Peripheral Nerve Sensitization" reflects potential peripheral mechanisms such as

7 induction of mechanosensitive sodium channels in the nerve sheath as a consequence

8 of focal inflammation [32]. Nerve mechanosensitivity to pressure and stretch in the

9 absence of nerve damage has been demonstrated in animal nerve inflammation

10 models $[33,34]$, and can be observed clinically in patients with radiating arm pain

11 [35] or leg pain [36]. The term "Peripheral Nerve Sensitization" describes a pain state

12 with marked nerve mechanosensitivity in the absence of neuropathic pain and

13 denervation.

15 Group M consists of subjects with a LANSS score $<12$, without marked neurological

16 deficits and negative nerve provocation tests. These clinical features indicate

17 "pseudoradicular" or somatic referred pain, as neural involvement in the subjects'

18 pain is unlikely. The main mechanism for somatic referred pain is convergence, where

19 afferent nerve fibers from the leg and from structures in the lower back converge upon

20 the same viscerosomatic neurons in the dorsal horn of the spinal cord [37].

\section{Examiners}

22 Two examiners (AS and KL), trained simultaneously by RR in the use of the QST

23 equipment, carried out all QST testing. The QST examiners were blinded to the

24 results of the physical examination. 
2 QST data that were not normally distributed were transformed logarithmically before

3 statistical analysis. The numbers of paradoxical heat sensations during the thermal

4 sensory limen procedure, cold pain thresholds, heat pain thresholds and vibration

5 detection thresholds were normally distributed as raw data. All other QST parameters

6 were normally distributed after logarithmical transformation.

7 To facilitate comparisons between parameters originally measured in different units,

8 normalized data for each of the QST parameters were converted to z-scores using

9 means and SDs from the control group $\left(\mathrm{z}\right.$-score $=$ Score $_{\text {single patient }}-$ Mean $_{\text {controls }} /$

$10 \mathrm{SD}_{\text {controls }}$ ) [17]. A $\mathrm{z}$-score of zero characterizes a value matching the group mean of

11 the healthy control subjects. Positive z-scores indicate a gain of function where the

12 patient is more sensitive to the tested stimulus compared to controls (hyperalgesia,

13 allodynia, hyperpathia) and negative z-scores indicate the patient has a loss of

14 sensation (hypoesthesia) compared to controls.

15

16 One-way ANOVAs and Chi square tests were used to analyze the difference in

17 general measures between groups (Table 1).

18 Two way ANOVAs were conducted for each QST parameter to test interaction effects

19 of group with body region and between subject main effects (group). The aim was to

20 investigate relationships of QST data with diagnostic groups. Where main effects or

21 interactions were significant, Tukey HSD post hoc tests were used to control for

22 multiple testing. All QST data are presented as Z-scores (mean \pm SEM) unless

23 otherwise indicated. SPSS version 17 (SPSS Inc., Chicago, USA) was used for

24 statistical analysis. 
1 3. Results

2 Subjects

3 Subjects had a mean age of 48 years and 39\% were men. Age, gender, pain duration

4 and proportion of patients with pain below the knee were comparable between groups

$5 \quad(p>0.50)$. Subjects with a score of 12 or more on the LANSS scale [27] were

6 classified as Neuropathic Sensitization $(n=20)$. The remaining symptomatic subjects

$7 \quad(\mathrm{n}=57)$ who had a LANSS scale score of less than 12 plus negative signs such as

8 hypoesthesia, muscle weakness or hyporeflexia were classified as Denervation

$9(\mathrm{n}=28)$. Of the remaining 29 symptomatic subjects, 9 exhibited positive neural

10 provocation tests, and were classified as Peripheral Nerve Sensitization. All other

11 subjects were classified as Musculoskeletal $(n=20)$ as there was no suggestion of

12 neural involvement (Figure 1). For detailed subject characteristics please see Table 1.

\section{QST findings}

14 Results showed relationships between QST data and diagnostic groups as there were

15 differences in QST parameters between groups across the tested body regions (group

16 main effect) (Table 2). All group main effects were between symptomatic subject

17 groups and the asymptomatic group. No significant differences were found between

18 the four symptomatic subject groups. Warm detection threshold was the only

19 parameter where the difference between groups varied significantly according to

20 region (significant group by region interaction), however no group main effects could

21 be detected for this parameter (Table 2). Allodynia was rare, there was one outlier

22 with severe allodynia over the back and paradoxical heat sensation was generally

23 more frequent at the affected foot for group Denervation, although these differences

24 were not significant at group level (Figure 3). Significant main effects for region 
1 across groups were not further analysed nor discussed, as these do not relate to the

2 research question.

3 QST procedures reveal differences between groups Neuropathic Sensitization,

4 Denervation and controls

5 The complete sensory profiles of the diagnostic groups Neuropathic Sensitization,

6 Denervation, Peripheral Nerve Sensitization, and Musculoskeletal over the foot,

7 lumbar spine, and dorsum of the hand are displayed in Figure 3. When comparing

8 symptomatic subject groups and asymptomatic controls, we found significant group

9 main effects for cold pain threshold, mechanical detection threshold, mechanical pain

10 threshold and mechanical pain sensitivity (Table 2).

12 Post hoc analysis with correction for multiple testing (Tukey HSD) for group main

13 effects revealed that group Neuropathic Sensitization had hyperalgesia to cold (CPT)

14 and to pinprick (MPT, MPS, all $\mathrm{p}<0.05$ ). Group Denervation also showed cold

15 hyperalgesia and in addition higher mechanical detection threshold indicating

16 mechanical hypaesthesia (MDT, $\mathrm{p}<0.05)$. For mean differences, $\mathrm{F}$ and $\mathrm{p}$ values,

17 please see Table 2.

\section{4. Discussion}

20 The results supported construct validity, as relationships between QST data and

21 diagnostic groups could be demonstrated. QST parameters differed between two

22 groups of subjects with leg pain and the group of asymptomatic subjects: Subjects in

23 group Neuropathic Sensitization showed marked signs of pain hypersensitivity, while

24 sensory deficits were most pronounced in group Denervation. The QST findings in 
1 these two groups match the presumed underlying pathomechanisms: Dominant

2 neuropathic pain mechanisms with sensory sensitization in group Neuropathic

3 Sensitization and mechanisms responsible for loss of conduction in group

4 Denervation. In contrast, groups Peripheral Nerve Sensitization and Musculoskeletal

5 were not significantly different to healthy controls across all QST parameters.

7 Decreased mechanical pain thresholds and cold hyperalgesia as observed in group

8 Neuropathic Sensitization are signs consistent with central sensitization [28]. Central

9 sensitization may arise as a result of a number of different mechanisms. Diminished

10 control of pain including cell death of inhibitory interneurons in the dorsal horn may

11 contribute to enhanced pain processing [28] as well as changed descending

12 modulatory mechanisms from the brain stem [38, 39]. Additionally, secondary

13 changes in cortical and subcortical brain regions, triggered by cognitions, emotions

14 and attention may further add to central sensitization and development of spontaneous

15 activity and pain [40, 41]. Another mechanism potentially contributing to

16 augmentation of central pain processing is deafferentation: Clinical and QST

17 examinations revealed deficits in large fibre function not only in group Denervation

18 but also in group Neuropathic Sensitization, indicating nerve fibre damage that for the

19 latter group may have induced secondary sensitization of higher order nociceptive

20 neurons [42]. QST findings from patients with other conditions thought to involve

21 central sensitization such as whiplash associated disorders [43], LBP [44] or

22 fibromyalgia [45] have also shown increased sensitivity to thermal and mechanical

23 pain stimuli consistent with findings in the present study. Central sensitization of the

24 nociceptive system is one of the main mechanisms contributing to neuropathic pain $25[46]$. 
2 Increased mechanical detection thresholds were found in group Denervation when

3 compared to healthy controls, this was most pronounced over the foot. Additionally,

4 although not significant, group Denervation showed the most pronounced deficits in

5 vibration, cold and warm detection over the foot (Fig. 3), consistent with a loss of

6 conduction. One possible explanation for the significantly elevated mechanical

7 detection threshold found in group Denervation could be mechanical compression of

8 the nerve root caused by prolapsed IVD tissue, osteophytes, facet joint hypertrophy or

9 ligamentum flavum hypertrophy [47]. Also chemical irritation of the nerve roots may

10 have similar effects. Proinflammatory cytokines such as tumor necrosis factor $\alpha$

11 released from nucleus pulposus cells or from inflamed arthritic facet joints can enter

12 the epidural space, contact nerve roots and thereby induce radicular symptoms with

13 large and small fibre deficits [48, 49].

15 A recent study [25] compared somatosensory profiles of subjects with somatic 16 referred pain $(n=15)$ with subjects with radicular pain $(n=12)$ and found that both 17 were significantly different to a healthy control group. The authors hypothesized that 18 mild root compression or an inflammatory perturbation of nerve roots in people with 19 pseudoradicular pain as well as in people with radicular pain may explain this

20 phenomenon. In contrast, the present study showed, in comparison to healthy

21 controls, no significant sensory dysfunction in groups Peripheral Nerve Sensitization

22 and Musculoskeletal, which are clinically comparable to patients with

23 "pseudoradicular symptoms". The reason for this may lie in a more differentiated 24 subclassification of subjects and consequently higher within group homogeneity. 
1 Some limitations should be pointed out. First of all, interaction effects between group

2 and body region could not be demonstrated. This indicates that only generalized

3 sensory changes over the entire body could be shown, but not localized changes. Also,

4 statistical analysis of QST data revealed significant differences only between two of

5 the four symptomatic groups and the asymptomatic group. This implies, firstly, that

6 construct validity could only be demonstrated for two of the groups, but not for the

7 classification as a whole. Secondly, the fact that no differences were found between

8 patient groups weakens conclusions in regard to construct validity. One possible

9 explanation is that group Peripheral Nerve Sensitization was unexpectedly small with

10 higher standard errors as a result. In addition, it is well known that psychosocial

11 factors such as hypervigilance or catastrophizing significantly influence pain

12 perception, however data in this respect were not available for the present study.

\section{5. Conclusion}

14 The results of this study provide preliminary evidence for the construct validity for

15 two of the four groups used in the new classification system as significant differences

16 of QST determined sensory and pain thresholds in groups Neuropathic Sensitization

17 and Denervation when compared to a group of asymptomatic subjects were shown.

18 These differences match presumed underlying mechanisms: Sensory deficits in group

19 Denervation and pain hypersensitivity in group Neuropathic Sensitization. Future

20 research should include assessment of further psychosocial covariates such as

21 catastrophizing or hypervigilance and focus on achieving equal group sizes. 


\section{References}

[1] Selim AJ, Ren XS, Fincke G, Deyo RA, Rogers W, Miller D, et al. The importance of radiating leg pain in assessing health outcomes among patients with low back pain. Results from the Veterans Health Study. Spine. 1998 Feb 15;23(4):470-4.

[2] Freynhagen R, Baron R, Gockel U, Tölle TR. Screening of neuropathic pain components in patients with chronic back pain associated with nerve compression: a prospective observational study (MIPORT) Curr Med Res Op. 2006;22:529 - 37.

[3] Ren XS, Selim AJ, Fincke G, Deyo RA, Linzer , Lee A, et al. Assessment of functional status, low back disability, and use of diagnostic imaging in patients with low back pain and radiating leg pain. J Clin Epidemiol. 1999 Nov;52(11):1063-71.

[4] Hill JC, Konstantinou K, Egbewale BE, Dunn KM, Lewis M, van der Windt D. Clinical Outcomes Among Low Back Pain Consulters With Referred Leg Pain In Primary Care. Spine (Phila Pa 1976). 2011 Feb 24;Epub ahead of print.

[5] Merskey H, Bogduk N. Classification of Chronic Pain. 2nd ed. Merskey H, Bogduk N, editors. Seattle: IASP Press; 1994.

[6] O'Neill CW, Kurgansky ME, Derby R, Ryan DP. Disc stimulation and patterns of referred pain. Spine. 2002;27(24):2776-81.

[7] Travell JG, Simons DG. The Lower Extremities. Philadelphia: Lippincott Williams \& Wilkins; 1983.

[8] Schwarzer AC, Aprill CN, Derby R, Fortin J, Kine G, Bogduk N. Clinical features of patients with pain stemming from the lumbar zygapophysial joints. Is the lumbar facet syndrome a clinical entity? Spine. 1994 May 15;19(10):1132-7.

[9] Vroomen PC, de Krom MC, Slofstra PD, Knottnerus JA. Conservative treatment of sciatica: a systematic review. J Spinal Disord. 2000 Dec;13(6):463-9.

[10] Luijsterburg PA, Verhagen AP, Ostelo RW, van Os TA, Peul WC, Koes BW. Effectiveness of conservative treatments for the lumbosacral radicular syndrome: a systematic review. Eur Spine J. 2007 Jul;16(7):881-99.

[11] Woolf CJ. Dissecting out mechanisms responsible for peripheral neuropathic pain: implications for diagnosis and therapy. Life Sci. 2004 Apr 9;74(21):2605-10.

[12] Schäfer A, Hall T, Briffa K. Classification of low back-related leg pain - A proposed patho-mechanism-based approach. Man Ther. 2009 Dec 31;14(2):222-30.

[13] Elvey RL, Hall TM. Neural tissue evaluation and treatment. In: Donatelli R, editor. Physical Therapy of the Shoulder. 3rd ed. New York ; Philadelphia: Churchill Livingstone; 1997. p. 131-52. 
[14] Schäfer A, Hall T, Lüdtke K, Mallwitz J, Briffa K. Interrater reliability of a new classification system for patients with low back related leg pain. J Man Manip Ther. 2009;17(2):109-17.

[15] Schäfer A, Hall T, Müller G, Briffa K. Outcomes differ between subgroups of patients with low back and leg pain following neural manual therapy: a prospective cohort study. Eur Spine J. 2011 Mar;20(3):482-90.

[16] de Vet HC, Terwee CB, Mokkink LB, Knol DL. Validity. Measurement in Medicine. Cambridge: Cambridge University Press; 2011. p. 150-201.

[17] Rolke R, Magerl W, Campbell KA, Schalber C, Caspari S, Birklein F, et al. Quantitative sensory testing: A comprehensive protocol for clinical trials. European Journal of Pain. 2006;10(1):77-88.

[18] Hansson P, Backonja M, Bouhassira D. Usefulness and limitations of quantitative sensory testing: clinical and research application in neuropathic pain states. Pain. 2007 Jun;129(3):256-9.

[19] Maier C, Baron R, Tolle TR, Binder A, Birbaumer N, Birklein F, et al. Quantitative sensory testing in the German Research Network on Neuropathic Pain (DFNS): somatosensory abnormalities in 1236 patients with different neuropathic pain syndromes. Pain. 2010 Sep;150(3):439-50.

[20] Rolke R, Baron R, Maier C, Tolle TR, Treede RD, Beyer A, et al. Quantitative sensory testing in the German Research Network on Neuropathic Pain (DFNS): standardized protocol and reference values. Pain. 2006 Aug;123(3):231-43.

[21] Geber C, Klein T, Azad S, Birklein F, Gierthmuhlen J, Huge V, et al. Test-retest and interobserver reliability of quantitative sensory testing according to the protocol of the German Research Network on Neuropathic Pain (DFNS): A multi-centre study. Pain. 2011 Mar;152(3):548-56.

[22] Felix ER, Widerstrom-Noga EG. Reliability and validity of quantitative sensory testing in persons with spinal cord injury and neuropathic pain. J Rehabil Res Dev. 2009;46(1):69-83.

[23] Scherens A, Maier C, Haussleiter IS, Schwenkreis P, Vlckova-Moravcova E, Baron R, et al. Painful or painless lower limb dysesthesias are highly predictive of peripheral neuropathy: Comparison of different diagnostic modalities. Eur J Pain. 2008 Sep 11.

[24] Selim MM, Wendelschafer-Crabb G, Hodges JS, Simone DA, Foster SX, Vanhove GF, et al. Variation in quantitative sensory testing and epidermal nerve fiber density in repeated measurements. Pain. 2010 Dec;151(3):575-81.

[25] Freynhagen R, Rolke R, Baron R, Tölle TR, Rutjes AK, Schu S, et al. Pseudoradicular and radicular low-back pain - A disease continuum rather than different entities? Answers from quantitative sensory testing. Pain. 2008 Jun $13 ; 135(1-2): 65-74$. 
[26] Sterling M, Jull G, Vicenzino B, Kenardy J. Sensory hypersensitivity occurs soon after whiplash injury and is associated with poor recovery. Pain. 2003

Aug;104(3):509-17.

[27] Bennett M. The LANSS Pain Scale: the Leeds assessment of neuropathic symptoms and signs. Pain. 2001 May;92(1-2):147-57.

[28] Woolf CJ, Mannion RJ. Neuropathic pain: aetiology, symptoms, mechanisms, and management. Lancet. 1999 Jun 5;353(9168):1959-64.

[29] Magerl W, Fuchs PN, Meyer RA, Treede RD. Roles of capsaicin-insensitive nociceptors in cutaneous pain and secondary hyperalgesia. Brain. $2001 \mathrm{Sep} ; 124(\mathrm{Pt}$ 9):1754-64.

[30] Treede RD, Handwerker HO, Baumgärtner U, Meyer RA, Magerl W. Hyperalgesia and Allodynia: Taxonomy, Assessment, and Mechanisms. In: Brune K, Handwerker HO, editors. Hyperalgesia: Molecular mechanisms and Clinical implications. Seattle: IASP Press; 2004. p. 3-15.

[31] Hall TM, Elvey RL. Management of mechanosensitivity of the nervous system in spinal pain syndromes. In: Boyling JD, Jull G, editors. Grieves Modern Manual Therapy. 3rd ed. Edinburgh: Churchill Livingstone; 2004. p. 413-33.

[32] Chen C, Cavanaugh JM, Song Z, Takebayashi T, Kallakuri S, Wooley PH. Effects of nucleus pulposus on nerve root neural activity, mechanosensitivity, axonal morphology, and sodium channel expression. Spine. 2004 Jan 1;29(1):17-25.

[33] Bove GM, Ransil BJ, Lin HC, Leem JG. Inflammation induces ectopic mechanical sensitivity in axons of nociceptors innervating deep tissues. $\mathbf{J}$ Neurophysiol. 2003 Sep;90(3):1949-55.

[34] Dilley A, Lynn B, Pang SJ. Pressure and stretch mechanosensitivity of peripheral nerve fibres following local inflammation of the nerve trunk. Pain. 2005

Oct;117(3):462-72.

[35] Allison GT, Nagy BM, Hall T. A randomized clinical trial of manual therapy for cervico-brachial pain syndrome -- a pilot study. Man Ther. 2002 May;7(2):95-102.

[36] Cleland JA, Childs JD, Palmer JA, Eberhart S. Slump stretching in the management of non-radicular low back pain: a pilot clinical trial. Man Ther. 2006 Nov;11(4):279-86.

[37] Jinkins JR. The anatomic and physiologic basis of local, referred and radiating lumbosacral pain syndromes related to disease of the spine. J Neuroradiol. 2004 Jun;31(3):163-80.

[38] Gardell LR, Vanderah TW, Gardell SE, Wang R, Ossipov MH, Lai J, et al. Enhanced evoked excitatory transmitter release in experimental neuropathy requires descending facilitation. J Neurosci. 2003 Sep 10;23(23):8370-9.

[39] Ren K, Dubner R. Descending modulation in persistent pain: an update. Pain. 2002 Nov;100(1-2):1-6. 
[40] Apkarian AV, Bushnell MC, Treede RD, Zubieta JK. Human brain mechanisms of pain perception and regulation in health and disease. Eur J Pain. 2005

Aug;9(4):463-84.

[41] Tracey I, Ploghaus A, Gati JS, Clare S, Smith S, Menon RS, et al. Imaging attentional modulation of pain in the periaqueductal gray in humans. J Neurosci. 2002 Apr 1;22(7):2748-52.

[42] Baumgärtner U, Magerl W, Klein T, Hopf HC, Treede RD. Neurogenic hyperalgesia versus painful hypoalgesia: two distinct mechanisms of neuropathic pain. Pain. 2002 Mar;96(1-2):141-51.

[43] Chien A, Eliav E, Sterling M. Hypoaesthesia occurs with sensory hypersensitivity in chronic whiplash - Further evidence of a neuropathic condition. Manual Therapy. 2009;14(2):138.

[44] Lewis C, Souvlis T, Sterling M. Sensory characteristics of tender points in the lower back. Manual Therapy. 2010;In Press, Corrected Proof.

[45] Staud R, Robinson ME, Price DD. Isometric exercise has opposite effects on central pain mechanisms in fibromyalgia patients compared to normal controls. Pain. 2005 Nov;118(1-2):176-84.

[46] Dickenson AH, Bee LA. Neurobiological mechanisms of neuropathic pain and its treatment. In: Castro-Lopes J, Raja S, Schmelz M, editors. Pain 2008 An updated review. Seattle: IASP press; 2008. p. 277-86.

[47] Kobayashi S, Baba H, Uchida K, Kokubo Y, Kubota C, Yamada S, et al. Effect of mechanical compression on the lumbar nerve root: localization and changes of intraradicular inflammatory cytokines, nitric oxide, and cyclooxygenase. Spine. 2005 Aug 1;30(15):1699-705.

[48] Tachihara H, Kikuchi S, Konno S, Sekiguchi M. Does facet joint inflammation induce radiculopathy?: an investigation using a rat model of lumbar facet joint inflammation. Spine. 2007 Feb 15;32(4):406-12.

[49] Olmarker K, Larsson K. Tumor necrosis factor alpha and nucleus-pulposusinduced nerve root injury. Spine. 1998 Dec 1;23(23):2538-44. 
Figure 1: Classification algorithm

LANSS $^{*}$ PAIN SCALE $\geq 12 ?$

- Paraesthesias

- Autonomous nerv. syst. changes

- Paroxysmal pain

- Burning pain

- Allodynia

- Pin prick hyper / hypoalgesia NO

NEUROLOGICAL DEFICIT?

At least two positive tests from at least two different domains: Reflexes, muscle power, pin prick and light touch
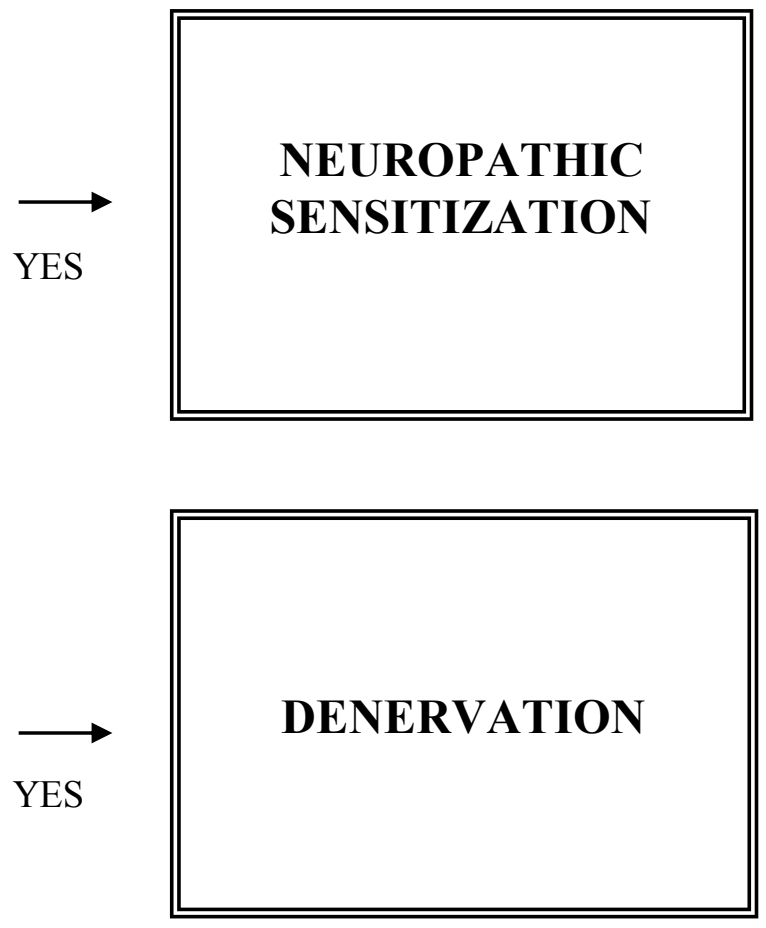

NO

NERVE TRUNK MECHANOSENSITIVITY?

Positive SLR or PKB and positive active flexion test in standing and positive nerve palpation

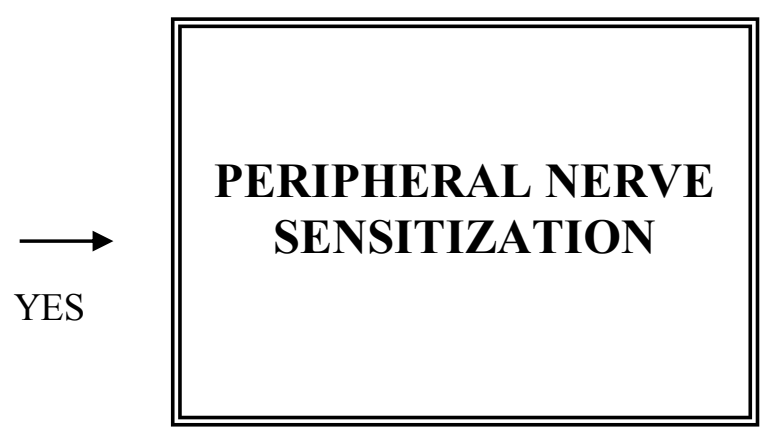

NO

MUSCULOSKELETAL

*LANSS: Leeds Assessment of Neuropathic Symptoms and Signs [27] 
Figure 2: Participant flow diagram

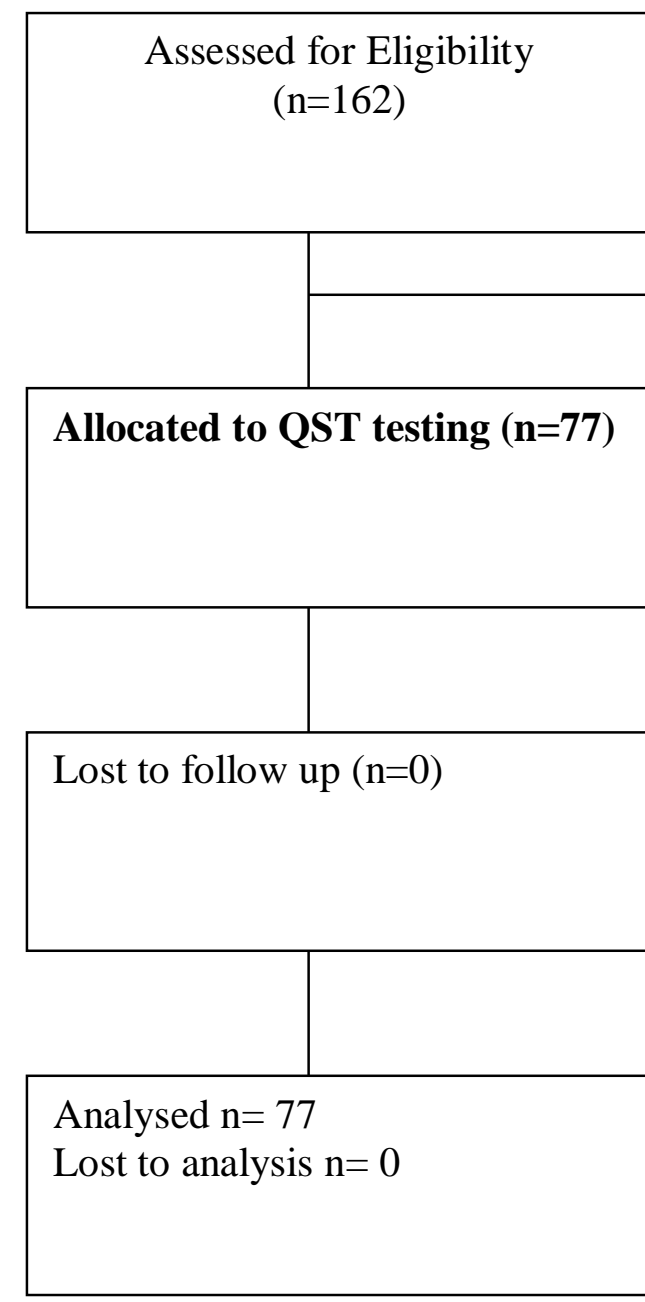

\section{Excluded $(\mathbf{n}=\mathbf{8 5})$}

Not meeting inclusion criteria

- Average pain rating below $3(n=23)$

- Bilateral leg pain $(n=15)$

- Rheumatoid arthritis $(n=5)$

- Diabetes $(n=4)$

Refused to participate $(n=26)$ 
Figure 3: QST profiles
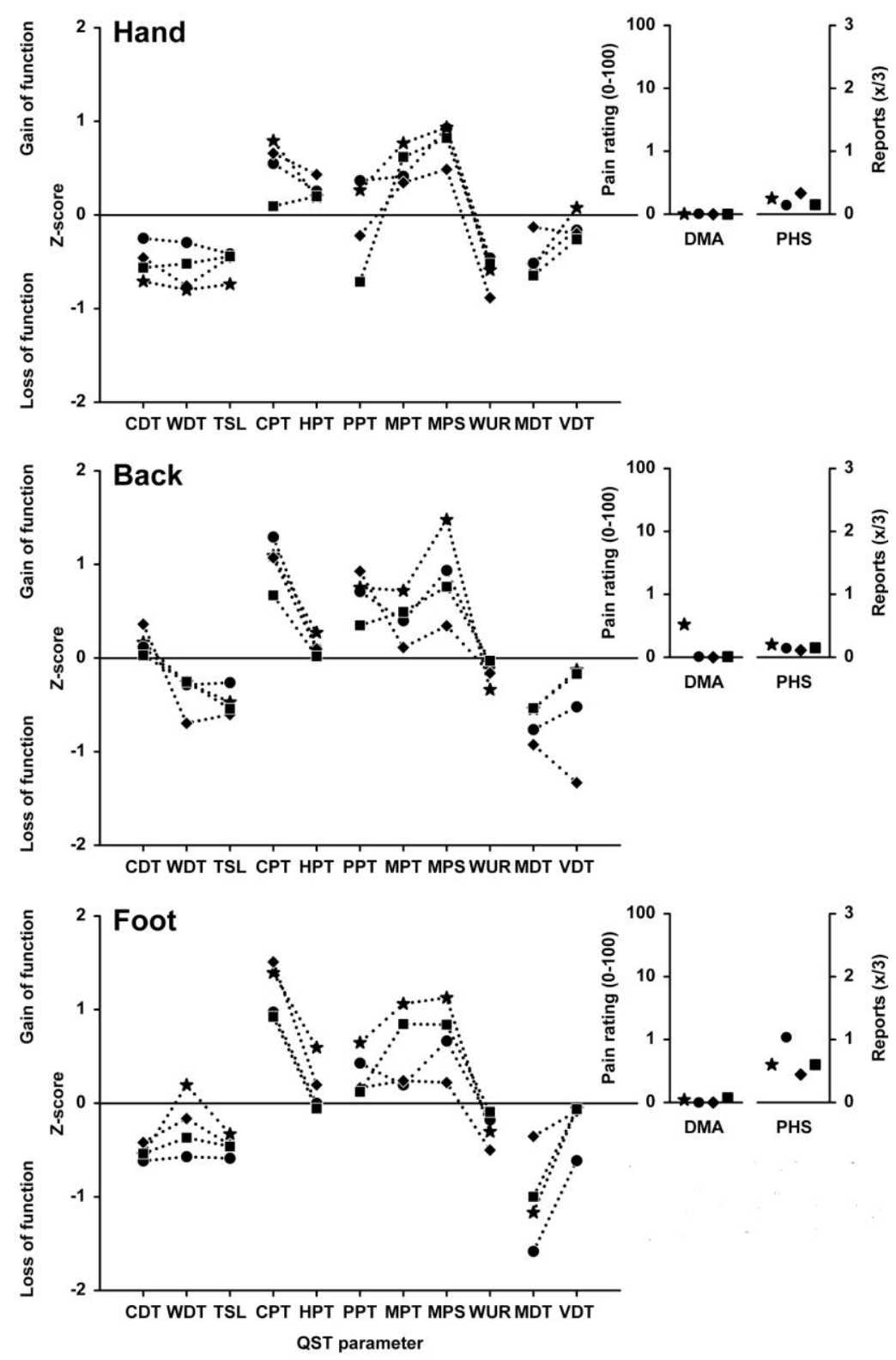

\footnotetext{
$\star \quad$ Group Neuropathic Sensitization

- Group Denervation

Group Peripheral Nerve Sensitization

Group Musculoskeletal
}

CDT, cold detection threshold; WDT, warm detection threshold; TSL, thermal sensory limen; CPT, cold pain threshold; HPT, heat pain threshold; PPT, pressure pain threshold; MPT, mechanical pain threshold; MPS, mechanical pain sensitivity; WUR, wind up ratio; MDT, mechanical detection threshold; VDT, vibration detection threshold; PHS, paradoxical heat sensation; DMA, dynamic mechanical allodynia.

The graphs in Figure 3 display the variance of group means (groups NS, D, PNS and M) from the mean of the asymptomatic controls in standardized units (z-scores). Positive z-scores indicate a gain of function, negative $\mathrm{z}$-scores indicate a loss of function. 
Table 1: Demographic and clinical data by diagnostic classification for subjects with LBRLP

\begin{tabular}{lllllll} 
& Total & $\begin{array}{l}\text { Neuropathic } \\
\text { Sensitization }\end{array}$ & Denervation & $\begin{array}{l}\text { Peripheral } \\
\text { Nerve } \\
\text { Sensitization }\end{array}$ & $\begin{array}{l}\text { Musculo- } \\
\text { skeletal }\end{array}$ & $p^{\text {p value }}$ \\
\hline n (\%) & 77 & $20(26)$ & $28(36)$ & $9(12)$ & $20(26)$ & \\
Age & $47.8(13.1)$ & $47.5(13.4)$ & $48.2(12.2)$ & $44.3(14.0)$ & $49.2(14.2)$ & $.83^{\mathrm{a}}$ \\
Gender (\% male) & 40 & 35 & 39 & 41 & 45 & $.92^{\mathrm{b}}$ \\
Pain below knee (\%) & 76.3 & 80.0 & 71.4 & 88.9 & 73.7 & $.71^{\mathrm{b}}$ \\
Pain duration (months) & $7.5(4.0)$ & $7.0(5.1)$ & $7.3(3.3)$ & $6.0(2.8)$ & $10.7(4.3)$ & $.76^{\mathrm{c}}$ \\
\end{tabular}

Values presented are means (Standard deviations) or percentage unless otherwise indicated

$\S$ Median (interquartile range)

${ }^{\text {a }}$ One-way ANOVA;

${ }^{\mathrm{b}} \chi^{2}$ test

${ }^{\mathrm{c}}$ Kruskall Wallis test 
Table 2: Statistics from two-way Analysis of variance comparing z-scores for QST parameters between four symptomatic groups and one asymptomatic group over different body regions

\begin{tabular}{|c|c|c|c|c|c|c|c|c|c|c|}
\hline $\begin{array}{l}\text { QST } \\
\text { parameter }\end{array}$ & $\begin{array}{l}\text { Group } \\
\text { main } \\
\text { effects }\end{array}$ & & $\begin{array}{l}\text { Tukey } \\
\text { HSD post } \\
\text { hoc for } \\
\text { group } \\
\text { main } \\
\text { effects }\end{array}$ & & & & $\begin{array}{l}\text { Body } \\
\text { region } \\
\text { main } \\
\text { effects }\end{array}$ & & $\begin{array}{l}\text { Inter- } \\
\text { action } \\
\text { region } \\
\text { by } \\
\text { group }\end{array}$ & \\
\hline & $\begin{array}{l}F \text { - } \\
\text { value }\end{array}$ & $\begin{array}{l}p- \\
\text { value }\end{array}$ & $\begin{array}{l}\text { Mean } \\
\text { difference } \\
\text { (z-score) }\end{array}$ & $\begin{array}{l}p \text { - } \\
\text { value }\end{array}$ & $\begin{array}{l}95 \% \\
\text { Confidence } \\
\text { Interval } \\
\text { upper } \\
\text { bound }\end{array}$ & $\begin{array}{l}\text { lower } \\
\text { bound }\end{array}$ & $\begin{array}{l}F \text { - } \\
\text { value }\end{array}$ & $\begin{array}{l}p \text { - } \\
\text { value }\end{array}$ & $\begin{array}{l}F \text { - } \\
\text { value }\end{array}$ & $\begin{array}{l}p- \\
\text { value }\end{array}$ \\
\hline CDT & 0.7 & 0.563 & & & & & 14.5 & 0.001 & 1.3 & 0.234 \\
\hline WDT & 1.1 & 0.374 & & & & & 2.9 & 0.062 & 2.1 & 0.042 \\
\hline TSL & 1.9 & 0.342 & & & & & 0.1 & 0.654 & 1.3 & 0.361 \\
\hline CPT & 3.3 & 0.015 & & & & & 7.5 & 0.001 & 1.0 & 0.428 \\
\hline C-NS & & & -1.09 & 0.019 & -2.06 & -0.12 & & & & \\
\hline C-D & & & -0.94 & 0.038 & -1.84 & -0.03 & & & & \\
\hline C-PNS & & & -1.08 & 0.108 & -2.3 & 0.14 & & & & \\
\hline C-M & & & -0.56 & 0.495 & -1.53 & 0.41 & & & & \\
\hline HPT & 0.5 & 0.891 & & & & & 0.6 & 0.845 & 0.9 & 0.531 \\
\hline MDT & 3.7 & 0.007 & & & & & 4.1 & 0.02 & 1.6 & 0.354 \\
\hline C-NS & & & 0.7509 & 0.058 & -0.012 & 1.52 & & & & \\
\hline C-D & & & .9543 & 0.003 & 0.24 & 1.67 & & & & \\
\hline C-PNS & & & 0.4689 & 0.659 & -0.5 & 1.43 & & & & \\
\hline C-M & & & 0.7188 & 0.078 & -0.05 & 1.49 & & & & \\
\hline MPT & 3.9 & 0.006 & & & & & 0.6 & 0.540 & 0.7 & 0.789 \\
\hline C-NS & & & -.8482 & 0.005 & -1.51 & -0.18 & & & & \\
\hline C-D & & & -0.3347 & 0.561 & -0.95 & 0.28 & & & & \\
\hline C-PNS & & & -0.2325 & 0.937 & -1.07 & 0.60 & & & & \\
\hline C-M & & & -0.652 & 0.058 & -1.32 & 0.01 & & & & \\
\hline MPS & 2.5 & 0.047 & & & & & 0.6 & 0.550 & 0.7 & 0.703 \\
\hline C-NS & & & -1.1798 & 0.033 & -2.29 & -0.06 & & & & \\
\hline C-D & & & -0.8415 & 0.168 & -1.88 & 0.2 & & & & \\
\hline C-PNS & & & -0.3507 & 0.957 & -1.75 & 1.05 & & & & \\
\hline C-M & & & -0.8075 & 0.267 & -1.92 & 0.31 & & & & \\
\hline WUR & 0.4 & 0.493 & & & & & 4.9 & 0.001 & 0.9 & 0.910 \\
\hline VDT & 1.3 & 0.260 & & & & & 2.6 & .083 & 1.6 & 0.131 \\
\hline PPT & 2.0 & 0.287 & & & & & 8.7 & 0.001 & 1.8 & 0.405 \\
\hline DMA & 1.0 & 0.745 & & & & & 1.5 & 0.734 & .7 & 0.820 \\
\hline PHS & 1.6 & 0.541 & & & & & 1.2 & 0.816 & .9 & 0.690 \\
\hline
\end{tabular}

CDT, cold detection threshold; WDT, warm detection threshold; TSL, thermal sensory limen; CPT, cold pain threshold; HPT, heat pain threshold; PPT, pressure pain threshold; MPT, mechanical pain threshold; MPS, mechanical pain sensitivity; WUR, wind up ratio; MDT, mechanical detection threshold; VDT, vibration detection threshold; PHS, paradoxical heat sensation; DMA, dynamic mechanical allodynia.

C- Control group

NS - Group Neuropathic Sensitization

D - Group Denervation

PNS - Group Peripheral Nerve Sensitization

M - Group Muskuloskeletal 